\title{
Predictive value of tumor-infiltrating lymphocytes to pathological complete response in neoadjuvant treated triple- negative breast cancers
}

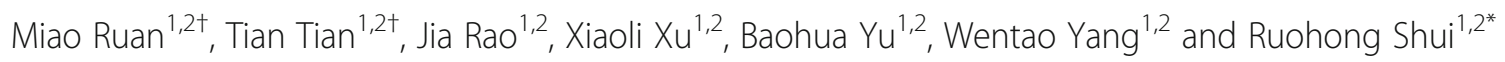

\begin{abstract}
Background: Triple-negative breast cancers (TNBCs) are a group of heterogeneous diseases with various morphology, prognosis, and treatment response. Therefore, it is important to identify valuable biomarkers to predict the therapeutic response and prognosis for TNBCs. Tumor-infiltrating lymphocytes (TILs) may have predictive value to pathological complete response (pCR) in neoadjuvant treated TNBCs. However, absence of standardized methodologies for TILs measurement has limited its evaluation and application in practice. In 2014, the International TILs Working Group formulated the recommendations of pathologic evaluation for TILs in breast cancers.

Methods: To evaluate the predictive value of TILs scored by methods recommended by International TILs Working Group 2014, we performed a retrospective study of TILs in 166 core needle biopsy specimens of primary invasive TNBCs with neoadjuvant chemotherapy (NAC) in a Chinese population. Intratumoral TILs (iTILs) and stromal TILs (sTILs) were scored respectively. The associations between TILs and PCR were analyzed.

Results: Both sTILs $(p=0.0001)$ and iTILs $(P=0.001)$ were associated with $\mathrm{pCR}$ in univariate logistic regression analysis. Multivariate logistic regression analysis indicated that both sTILs $(P=0.006)$ and $\mathrm{iTILS}(P=0.04)$ were independent predictors for $\mathrm{PCR}$. Receiver operating characteristics $(\mathrm{ROC})$ curve analysis was used to identify the optimal thresholds of TILs. TNBCs with more than $20 \%$ sTILs $(P=0.001)$ or with more than $10 \%$ iTILs $(P=0.003)$ were associated with higher pCR rates in univariate analysis. Multivariate analysis showed that a $20 \%$ threshold of sTILs $(P=0.005)$ was an independent predictive factor for $\mathrm{pCR}$.

Conclusions: Our study indicated that TILs scored by recommendations of International TILs Working Group 2014 in pre-NAC core needle biopsy specimens was significantly correlated with pCR in TNBCs, higher TILs scores predicting higher pCR rate. Both sTILs and iTILs were independent predictors for pCR in TNBCs. A 20\% threshold for sTILs may be feasible to predict PCR to NAC in TNBCs.
\end{abstract}

Keywords: Tumor-infiltrating lymphocytes, Breast cancer, Triple-negative, Pathological complete response, Predictive factor

\footnotetext{
* Correspondence: shuiruohong2014@163.com

${ }^{\dagger}$ Miao Ruan and Tian Tian contributed equally to this work.

${ }^{1}$ Department of Pathology, Fudan University Shanghai Cancer Center,

Shanghai, China

²Department of Oncology, Shanghai Medical College, Fudan University,

Shanghai, China
}

(c) The Author(s). 2018 Open Access This article is distributed under the terms of the Creative Commons Attribution 4.0 International License (http://creativecommons.org/licenses/by/4.0/), which permits unrestricted use, distribution, and reproduction in any medium, provided you give appropriate credit to the original author(s) and the source, provide a link to the Creative Commons license, and indicate if changes were made. The Creative Commons Public Domain Dedication waiver (http://creativecommons.org/publicdomain/zero/1.0/) applies to the data made available in this article, unless otherwise stated. 


\section{Background}

Triple-negative breast cancers (TNBCs) are defined as a group of breast cancer characterized by lacking of estrogen receptor (ER), progesterone receptor (PgR) and human epidermal growth factor receptor 2 (HER2) protein expression [1]. Most of TNBCs have higher risk of early distant recurrence, mortality and more aggressive clinical behavior compared with other subtypes of breast cancers [2, 3]. Owing to the absence of effective targeted therapy, chemotherapy is the only recommended systemic treatment for TNBCs at present stage [4]. However, various therapeutic strategies have been explored, among which immunotherapy may have potential benefits to treat TNBCs $[5,6]$. Therefore, several studies have been carried out to evaluate the predictive and prognostic values of tumor-infiltrating lymphocytes (TILs) in TNBCs, which have indicated that high levels of TILs may be associated with a better clinical outcome and a better response to chemotherapy in TNBCs [7-9].

It has been demonstrated that patients gaining pathological complete response ( $\mathrm{pCR}$ ) to neoadjuvant chemotherapy (NAC) may experience prolonged disease-free survival, especially in TNBCs $[3,7,8]$. Many biomarkers to predict pCR for NAC in TNBCs have been analyzed, such as immune-related gene signatures and clinicopathologic factors. Several studies have indicated that TILs in pre-NAC samples may be used to predict pCR in TNBCs [9-12]. However, methodologies of TILs evaluation in these studies were not standardized, which has hindered its application in clinical practice.

Lymphocyte-predominant breast cancer (LPBC), firstly proposed by Denkert et al., was defined as tumors with a particularly strong lymphocytic infiltration whether in tumor stroma or cell nests [9]. The LPBC-cutoff was generally $50 \%$ or $60 \%$ in previous literatures $[9,13]$. However, owing to the relatively low proportion of LPBC in routine practice, it may be unreasonable to define $50-60 \%$ as the threshold for LPBC.

In this study, we conducted a retrospective analysis of TILs in 166 core needle biopsy specimens of TNBCs with NAC in a Chinese population. Stromal TILs (sTILs) and intratumoral TILs (iTILs) in pre-NAC specimens were scored using the method recommended by the International TILs Working Group 2014 [14], and the correlation between TILs and neoadjuvant chemotherapy response was analyzed. The optimal thresholds of TILs to predict pCR in TNBCs were explored. The aim of our study was to examine the predictive value of TILs to $\mathrm{pCR}$ in neoadjuvant treated TNBCs, and to evaluate the feasibility of the scoring methods in clinical practice.

\section{Methods}

\section{Patients and samples}

166 consecutive core needle biopsy specimens of primary invasive TNBCs diagnosed and treated with NAC following up operation between 2011 and 2016 were extracted from the pathology database of Fudan University Shanghai Cancer Center. The inclusion criteria were as follows: primary invasive TNBCs; neoadjuvant therapy before surgical operation; available complete clinicopathologic data (age, tumor size, tumor grade, histological type, lymphovascular invasion, lymph node status, Miller-Payne grade, ER, PgR, HER2 and Ki-67 index). All specimens were fixed with $10 \%$ neutral phosphate-buffered formalin and paraffin-embedded. $4 \mu \mathrm{m}$-thick slices of representative tumor blocks were stained with hematoxylin and eosin (H\& E). Tumors were defined as triple negative as following: < 1\% of ER and PgR immunoreactivity, and absence of HER2 protein overexpression or gene amplification $[15,16]$.

\section{Pathologic evaluation}

All core needle biopsy specimens and surgical slices were reviewed by two experienced breast pathologists (R.S. and W.Y.) to confirm the histological type, according to 2012 World Health Organization (WHO) Classification of Tumours of the Breast [17]. Histological grade of tumor was evaluated in pre-NAC core needle biopsy specimens by the Nottingham grading system [18, 19]. Miller-Payne grading system was used to evaluate the pathological response in surgical specimens [20]. The pCR of chemotherapeutic response was the endpoint of our study, which was defined as the absence of invasive carcinoma in the breast tissue and axillary lymph nodes in surgical specimens.

Evaluation of TILs on core needle biopsy specimens was performed by two breast pathologists (M.R. and T.T.). The two observers were trained by the evaluation criteria recommended by the International TILs Working Group 2014, and scored each case independently in a blind manner. The mean values of two observers were obtained as final scores for each case. STILs were defined as the percentage of tumor stroma containing infiltrating lymphocytes and plasma cells, which should exclude polymorphonuclear leukocytes (Fig. 1a, b). ITILs were defined as the percentage of lymphocytes and plasma cells within tumor cell nests or in direct contact with the tumor cells (Fig. 1a, c). Areas of in situ carcinomas, normal lobules, necrosis, hyalinization and crush artifacts were not included [14]. The TILs were scored in an average value throughout full sections rather than hotspots. The results were scored in increments of 10; 0 was defined as < 5\%; 10 was defined as 5\% to $10 \%$; 20 was defined as $11 \%$ to $20 \%$ and all other scores were rounded up to the next highest decile (Fig. 1d-1g).

\section{Statistical analysis}

Two types of variables were used to test: one was continuous variables (per 10\% increment); the other was binary variables categorized by $20 \%$ score cutoff (sTILs) and $10 \%$ score cutoff (iTILs). The associations between 


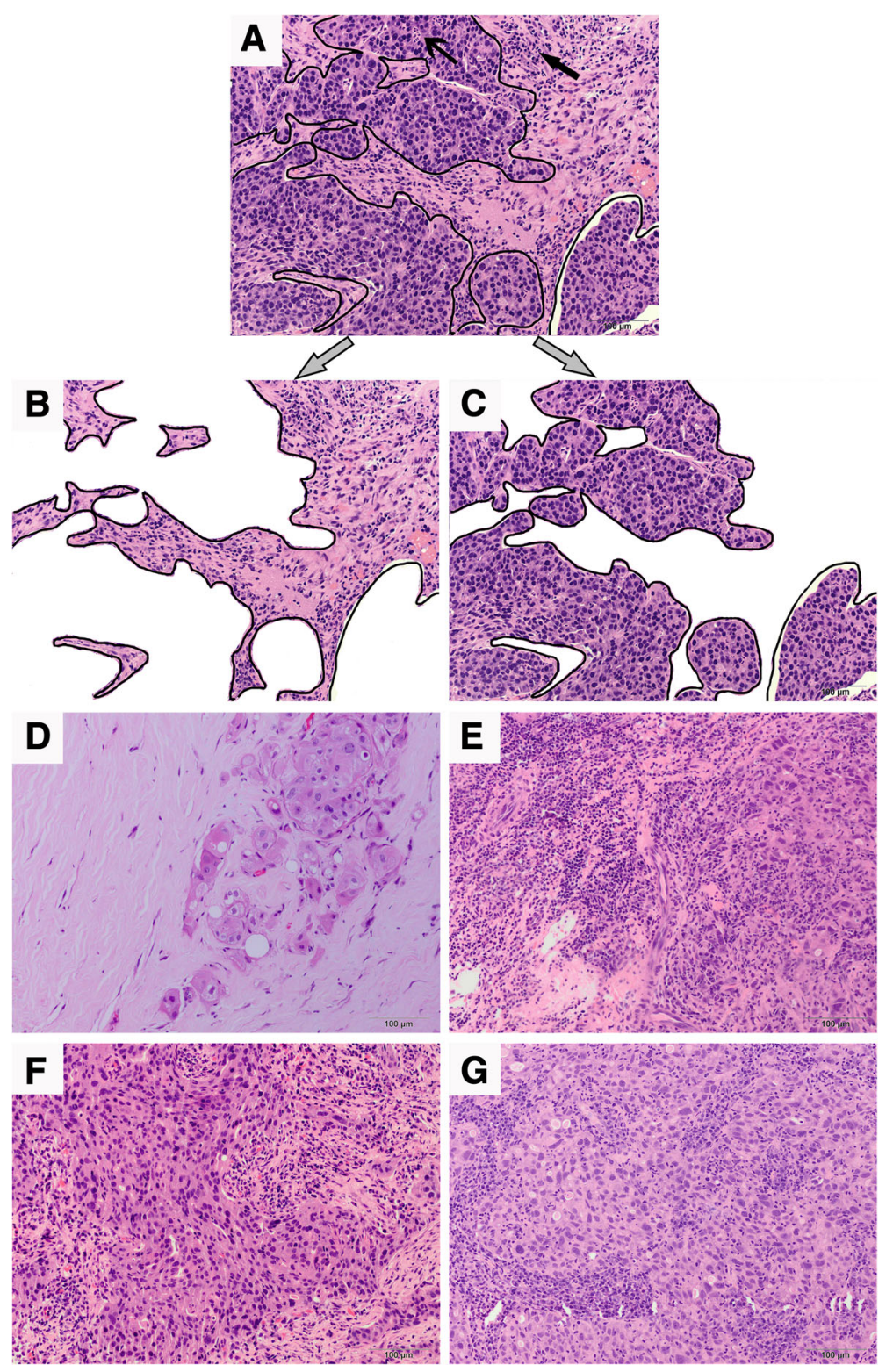

Fig. 1 Histopathologic evaluation of TILs and different scores of TILS in TNBCS. a: The areas of sTILs and iTILs evaluation were distinguished by black lines (bold black arrow pointed to plasma cells and lymphocytes, Fine black arrow pointed to polymorphonuclear leukocytes which should be excluded in TILs evaluation). b: The area of sTILs evaluation: 20\%; c: The area of iTILs evaluation: 0\%. d: sTILs: 0\%. e: sTILs: $50 \%$. f: iTILs: $10 \%$. g: iTILs: $20 \%$ (All $\times 200$ magnification)

TILs and clinicopathologic characteristics including patients' age ( $\leq 50$ years versus $>50$ years), tumor size $(\leq$ $2 \mathrm{~cm}, 2-5 \mathrm{~cm}$ versus $>5 \mathrm{~cm})$, tumor grade $(1,2$ versus 3$)$, lymph node status (negative versus positive), histological type (IDC versus special type), LVI (negative versus positive), Ki-67 index, Miller-Payne grade (1-5) and neoadjuvant chemotherapy regiments were analyzed. The correlations of TILs as continuous variables with polytomous variables were evaluated with Kruskal-Wallis test. Mann-Whitney test was performed to identify the associations between TILs and binary variables. The associations between TILs and continuous variables (Ki-67 index and Miller-Payne grade) were evaluated with Spearman's rank correlation analysis ( $r$ ). The intraclass correlation coefficient analysis was used to evaluate the interobserver agreement of sTILs and iTILs scores. The correlation between pCR after NAC and TILs was analyzed by univariate logistic regression analysis. Multivariate logistic regression analysis was used to identify the independent predictors for chemotherapeutic response. Stratified analysis was used to investigate the correlation between TILs and $\mathrm{pCR}$ across different clinicopathologic subgroups. 
Evaluation of heterogeneity effects and test for trend were performed by chi-squared test. Receiver operating characteristics (ROC) curve was conducted to detect the optimal thresholds of TILs and the predictive model to predict pCR. The maximum Youden's Index $(J=$ sensitivity + specificity - 1) was calculated to define the optimal thresholds, then univariate and multivariate regression analysis were used to evaluate the predictive value of TILs as binary variables for $\mathrm{pCR}$. A two-side $p$-value $<0.05$ was considered statistically significant. All statistical analyses were performed using the SPSS version 20.0 (SPSS Inc., Chicago, IL) and STATA version 13.1 (Stata Corporation, College Station, TX, USA).

\section{Results}

\section{Clinicopathologic characteristics}

The clinicopathologic characteristics of 166 TNBCs were listed in Table 1 . The age of patients ranged from 25 to 77 years with a mean age of 50 years. All patients were female. The pre-NAC tumor size was assessed according to radiology findings. The tumor grade was estimated in the pre-NAC specimens of core needle biopsy. In core needle biopsy, 164 (98.8\%) cases were diagnosed as invasive ductal carcinoma of no special type (IDC) and only $2(1.2 \%)$ cases were invasive carcinoma with special subtypes (carcinoma with apocrine differentiation in 2 cases). Lymph nodes involvement was found in 113 (68.1\%) cases by fine needle aspiration (FNA) before neoadjuvant treatment. Patients underwent NAC based on the combination treatment regimen of anthracycline and paclitaxel $(136 / 166,81.9 \%)$, or paclitaxel and platinum $(30 / 166,18.1 \%)$. All patients received surgical operation after eight cycles of NAC. 141 (84.9\%) patients underwent mastectomy, and breast-conserving surgery was performed in $25(15.1 \%)$ patients. Lymphovascular invasion (LVI) was observed in the postoperative slices of $36(21.7 \%)$ cases. 67 (40.4\%) cases obtained pCR, and non-pCR were observed in 99 (59.6\%) cases.

\section{Correlations between TILs and clinicopathologic parameters}

The distribution of TILs in 166 core needle biopsy specimens was summarized in Table 2. The average score of sTILs was $15 \%$ (range: $0-60 \%$ ), and the average score of iTILs was 5\% (range: $0-30 \%$ ). STILs score was positively associated with iTILs $(r=0.65, p<0.001)$ by Spearman correlation analysis. Using the intraclass correlation coefficient (ICC) analysis, the interobserver agreement of sTILs and iTILs assessment both were excellent (sTILs: ICC $0.91,95 \%$ CI $0.88-0.93, P=0.001$; iTILs: ICC $0.83,95 \%$ CI 0.77-0.88, $P=0.001$ ).

The correlations between clinicopathologic characteristics and TILs were analyzed in Table 1 . Mann-Whitney test showed that both sTILs $(P=0.004)$ and iTILs $(P=$
0.03 ) were positively associated with histological grade (Fig. 2a, b), tumors with grade 3 having more lymphocytic infiltration than grade 2. ITILs were positively correlated with negative lymphovascular invasion $(P=0.03$, Fig. $2 \mathrm{c})$. Spearman's rank correlation analysis revealed that sTILs were positively correlated with Miller-Payne grade, higher sTILs scores in pre-NAC specimens having higher Miller-Payne grade after operation $(r=0.263, P=0.001$, Fig. 2d). Spearman's rank correlation analysis showed that both higher scores of sTILs $(r=0.236, P=0.002)$ and iTILs $(r=0.346, P=0.001)$ were positively related with higher Ki-67 index in TNBCs (Fig. 2e, f). There was no significant association of TILs with patients' age, tumor size, lymph node status, histological type and neoadjuvant chemotherapy regimens.

\section{Correlations between TILs and $\mathrm{pCR}$}

The relationship between TILs and pCR was analyzed by logistic regression analysis (Table 3). TILs were scored as continuous variables (per 10\% increment). Univariate analysis showed that both sTILs (per 10\% sTILs: OR 1.07, 95\% CI 1.03-1.10, $P=0.0001$ ) and iTILs (per 10\% iTILs: OR 1.10, 95\% CI 1.04-1.16, $P=0.001$ ) were significantly correlated with pCR. Higher TILs scores indicated higher $\mathrm{pCR}$ rate. Multivariate analysis demonstrated that both sTILs (per 10\% sTILs: OR 1.05, 95\% CI $1.02-1.09, P=0.006$ ) and iTILs (per 10\% iTILs: OR 1.06, 95\% CI 1.00-1.12, $P=0.04$ ) were independent predictors for pCR, irrespective of other clinicopathologic factors.

Stratified analysis was used to investigate whether the predictive value of TILs might be different in every subgroup of clinicopathologic characteristics (Table 4). The chi-squared test showed that there was no significant difference for TILs predicting the rate of $\mathrm{pCR}$ in each subgroup $(p>0.05)$.

\section{The optimal thresholds of TILs to predict $\mathrm{pCR}$}

Receiver operating characteristics (ROC) curve analysis was used to identify the optimal thresholds of TILs distinguishing pCR from non-pCR cases (Fig. 3). It was revealed that the area under the curve (AUC) of sTILs level was 0.645 (95\% CI $0.575-0.747, P=0.0001$ ) and the best cutoff value of sTILs to predict pCR was 15\%. The AUC of iTILs level was $0.612(95 \%$ CI $0.542-0.717, P=0.005)$ and the best cutoff value of iTILs was $5 \%$. Because TILs was scored in $10 \%$ increments in our study, all cases were categorized into two groups respectively according to the results of ROC curve: TNBCs with sTILs $\geq 20 \%$ and TNBCs with sTILs $<20 \%$; TNBCs with iTILs $\geq 10 \%$ and TNBCs with iTILs $<10 \%$. The sensitivity, specificity, positive predictive value (PPV), negative predictive value (NPV), accuracy and Youden's Index of the 20\% threshold for sTILs and the $10 \%$ for iTILs to predict pCR were showed in Table 5. 
Table 1 Correlations between TILs and clinicopathologic characteristics in TNBCs

\begin{tabular}{|c|c|c|c|}
\hline Characteristics & No. of patients (\%) & $P$-value of sTILs & $P$-value of iTILs \\
\hline \multicolumn{4}{|l|}{ Age (years) } \\
\hline$<50$ & $80(48.2)$ & $0.87^{\mathrm{a}}$ & $0.79^{\mathrm{a}}$ \\
\hline$\geq 50$ & $86(51.8)$ & & \\
\hline \multicolumn{4}{|l|}{ Tumor size $(\mathrm{cm})$} \\
\hline$\leq 2$ & $22(13.2)$ & $0.88^{\mathrm{b}}$ & $0.16^{\mathrm{b}}$ \\
\hline $2-5$ & $115(69.3)$ & & \\
\hline$>5$ & $29(17.5)$ & & \\
\hline \multicolumn{4}{|l|}{ Lymph node status } \\
\hline Negative & $53(31.9)$ & $0.78^{\mathrm{a}}$ & $0.23^{\mathrm{a}}$ \\
\hline Positive & $113(68.1)$ & & \\
\hline \multicolumn{4}{|l|}{ Histological type } \\
\hline IDC & $164(98.8)$ & $0.58^{\mathrm{a}}$ & $0.33^{\mathrm{a}}$ \\
\hline Special type & $2(1.2)$ & & \\
\hline \multicolumn{4}{|l|}{ Histological grade } \\
\hline 2 & $31(18.7)$ & $0.004^{a+}$ & $0.03^{a+}$ \\
\hline 3 & $135(81.3)$ & & \\
\hline \multicolumn{4}{|l|}{ LVI } \\
\hline Negative & $130(78.3)$ & $0.17^{\mathrm{a}}$ & $0.03^{a+}$ \\
\hline Positive & $36(21.7)$ & & \\
\hline \multicolumn{4}{|l|}{ Miller-Payne grade } \\
\hline 1 & $17(10.2)$ & $0.001^{\mathrm{b}+}$ & $0.06^{\mathrm{b}}$ \\
\hline 2 & $26(15.7)$ & & \\
\hline 3 & $32(19.3)$ & & \\
\hline 4 & $24(14.4)$ & & \\
\hline 5 & $67(40.4)$ & & \\
\hline \multicolumn{4}{|l|}{ NAC } \\
\hline $\begin{array}{l}\text { Anthracycline + } \\
\text { paclitaxel }\end{array}$ & $136(81.9)$ & $0.98^{\mathrm{a}}$ & $0.99^{\mathrm{a}}$ \\
\hline $\begin{array}{l}\text { Paclitaxel } \\
+ \text { platinum }\end{array}$ & $30(18.1)$ & & \\
\hline
\end{tabular}

Abbreviations: TNBCs triple-negative breast cancers, sTILs stromal tumorinfiltrating lymphocytes, iTILs intratumoral tumor-infiltrating lymphocytes, IDC invasive ductal carcinoma of no special type, LVI lymphovascular invasion, NAC neoadjuvant chemotherapy

${ }^{a}$ Mann-Whitney test

${ }^{b}$ Kruskal-Wallis test

+The $p$ value is significant

Meanwhile, 20\% threshold of sTILs was compared with $50 \%$ and $60 \%$ thresholds. The sensitivity, specificity, PPV, NPV, accuracy and Youden's Index of these thresholds for sTILs were summarized in Table 5. It was shown that $41.6 \%$ of patients had a level of sTILs more than $20 \%$, and the sensitivity and specificity were higher than other thresholds. 10\% threshold of iTILs was also compared with $20 \%$ and $30 \%$ thresholds (Table 5). $42.8 \%$ of patients had a level of iTILs more than $10 \%$, and the sensitivity and specificity were higher than other thresholds. Therefore, our study indicated that $20 \%$ threshold
Table 2 The distribution of TILs scores in TNBCs

\begin{tabular}{lll}
\hline Score (\%) & Cancer with sTILs No. (\%) & Cancer with iTILs No. (\%) \\
\hline 0 & $17(10.2)$ & $95(57.2)$ \\
10 & $80(48.2)$ & $64(38.6)$ \\
20 & $41(24.7)$ & $5(3)$ \\
30 & $21(12.7)$ & $2(1.2)$ \\
40 & 0 & 0 \\
50 & $4(2.4)$ & 0 \\
60 & $3(1.8)$ & 0 \\
70 & 0 & 0 \\
80 & 0 & 0 \\
90 & 0 & 0 \\
100 & 0 & 0 \\
\hline
\end{tabular}

Abbreviations: TNBCs triple-negative breast cancers, sTILs stromal tumorinfiltrating lymphocytes, iTLL intratumoral tumor-infiltrating lymphocytes

of sTILs and $10 \%$ threshold of iTILs may be optimal to predict $\mathrm{pCR}$ in TNBCs.

\section{Predictive value of the optimal thresholds of TILs}

Logistic regression analysis was performed to evaluate the predictive value of the optimal thresholds in our study. As shown in Table 6, TNBCs with more than $20 \%$ sTILs (OR 2.87, 95\% CI 1.51-5.47, $P=0.001$ ) or more than $10 \%$ iTILs (OR 2.62, 95\% CI 1.38-4.97, $P=0.003$ ) both were significantly associated with higher $\mathrm{pCR}$ rate in univariate analysis. Multivariate analysis confirmed that a $20 \%$ threshold of sTILs (OR 2.85, 95\% CI 1.38 5.90, $P=0.005$ ) was an independent predictive factor for pCR, while a $10 \%$ threshold of iTILs wasn't an independent predictive factor for $\mathrm{pCR}(P>0.05)$.

In view of the relatively low sensitivity and specificity of the $20 \%$ threshold for sTILs to independently predict pCR (56.7\% and $68.7 \%$, respectively), a predictive model for response to NAC was performed by combining sTILs with clinicopathologic parameters which had a significant association with the rate of $\mathrm{pCR}$ in univariate analysis (including patients' age, histological grade, LVI and Ki-67 index) (Table 6). ROC curve analysis demonstrated that the AUC for the combination of these five variables was 0.785 (95\% CI $0.714-0.856, P=0.0001$ ), and the sensitivity and specificity to predict pCR were $77.6 \%$ and $72.7 \%$, respectively. And it's indicated that the combined predictive model may be more optimal to predict PCR than these clinicopathologic parameters alone in TNBCs (Fig. 4).

\section{Discussion}

TNBCs are a group of heterogeneous diseases with various morphology, prognosis, and treatment response. Therefore, it is important to identify valuable biomarkers to predict the therapeutic response and prognosis for TNBCs. Several studies have demonstrated the prognosis 

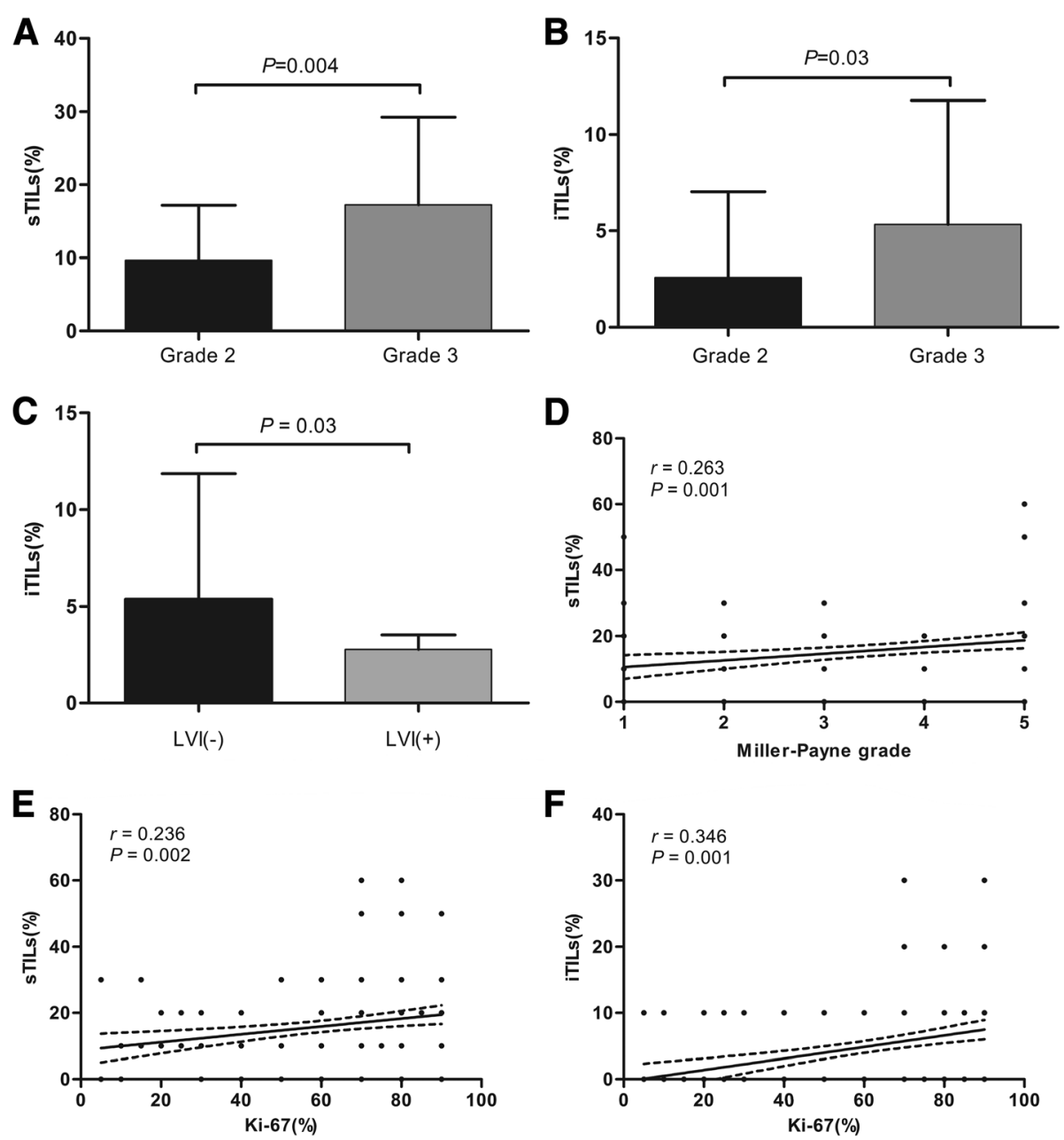

Fig. 2 The correlations of TILs with histological grade, LVI, Miller-Payne grade and Ki-67 index in TNBCs. Y axis represented the scores of TILs; X axis represented histological grade (2 or 3), LVI (-/+), Miller-Payne grade (1-5) or Ki-67 index (\%). a and $\mathbf{b}$ : Both sTILs and iTILs were positively associated with histological grade (sTILs: $P=0.004$; iTILs: $P=0.03$ ). c: ITILs were positively correlated with negative lymphovascular invasion $(P=$ 0.03). Dots corresponded to the scores of TILs and whiskers corresponded to its Standard Error. Red line corresponded to the mean scores of TILS. d: STILs was positively correlated with Miller-Payne grade $(r=0.263, P=0.001)$. e and $\mathbf{f}$ : STILs and iTILs scores were positively associated with Ki-67 index (sTILs: $r=0.236, P=0.002 ;$ iTILs: $r=0.346, P=0.001$ ). LVI: Iymphovascular invasion

and the predictive values of TILs in TNBCs [12, 21-24]. However, absence of standardized methodologies for TILs measurement has limited its evaluation and application in practice. In 2014, the International TILs Working Group formulated the recommendations of pathologic evaluation for TILs in breast cancers. The recommendations need to be further validated in multiple laboratories before application in routine practice. The study of Pruneri et al. supported the validity of TILs evaluation recommendations in the clinical practice, indicating that each $10 \%$ increase in TILs strongly predicted better survival in TNBCs [25]. However, Park et al's study showed that TILs scored by the recommendations may not be useful for predicting survival outcomes in early TNBCs [26]. In our previous study, we carried out a retrospective analysis of TILs in 425 primary invasive TNBCs using the recommendations, indicating that TILs scored by the recommendations could be associated with the prognosis of TNBCs [27]. In this study, we performed a retrospective analysis of TILs in 166 core needle biopsy specimens of TNBCs with NAC, aimed to evaluate the predictive value of TILs scored by the recommendations to PCR. Our study indicated that TILs scored by the recommendations in pre-NAC core needle biopsy of TNBCs were significantly correlated with $\mathrm{pCR}$, higher TILs score strongly predicting higher pCR rate, and TILs score was an independent predictor for pCR. Another issue which should be evaluated is the reproducibility of the recommendations. Swisher et al. evaluated the interobserver agreement of TILs scored by recommendations among four observers, and showed an acceptable agreement in TILs evaluation [28]. In our study, an excellent interobserver agreement between two observers was 
Table 3 Correlations between TILs and pCR in neoadjuvant treated TNBCs

\begin{tabular}{|c|c|c|c|c|c|c|}
\hline \multirow[t]{2}{*}{ Variables } & \multicolumn{3}{|c|}{ Univariate analysis } & \multicolumn{3}{|c|}{ Multivariate analysis } \\
\hline & $\mathrm{OR}$ & $95 \% \mathrm{Cl}$ & $P$-value & $\mathrm{OR}$ & $95 \% \mathrm{Cl}$ & $P$-value \\
\hline ITILs (per 10\%) & 1.10 & $1.04-1.16$ & $0.001^{*}$ & 1.06 & $1.00-1.12$ & $0.04^{*}$ \\
\hline STILs (per 10\%) & 1.07 & $1.03-1.10$ & $0.0001^{*}$ & 1.05 & $1.02-1.09$ & $0.006^{*}$ \\
\hline Age (years) (<50 vs. $\geq 50$ ) & 0.51 & $0.27-0.95$ & $0.04^{*}$ & 0.65 & $0.31-1.35$ & 0.25 \\
\hline Histological grade (2 vs. 3) & 2.74 & $1.11-6.80$ & $0.03^{*}$ & 1.02 & $0.34-3.08$ & 0.98 \\
\hline Tumor size $(\mathrm{cm})(\leq 2$ vs. $2-5$ vs. $>5)$ & 1.30 & $0.74-2.29$ & 0.37 & 1.30 & $0.68-2.47$ & 0.43 \\
\hline Nodal status (negative vs. positive) & 0.93 & $0.48-1.81$ & 0.84 & 1.52 & $0.72-3.22$ & 0.28 \\
\hline LVI (negative vs. positive) & 0.06 & $0.01-0.26$ & $0.0001^{*}$ & 0.07 & $0.02-0.29$ & $0.0001^{*}$ \\
\hline Ki-67 index & 1.03 & $1.01-1.04$ & $0.001^{*}$ & 1.02 & $1.006-1.04$ & $0.009^{*}$ \\
\hline NAC (Anthracycline + paclitaxel vs. paclitaxel + platinum) & 0.83 & $0.37-1.87$ & 0.65 & 1.10 & $0.41-2.94$ & 0.85 \\
\hline
\end{tabular}

Abbreviations: $p C R$ pathological complete response, TNBCs triple-negative breast cancers, sTILs stromal tumor-infiltrating lymphocytes, iTILs intratumoral tumorinfiltrating lymphocytes, LVI lymphovascular invasion, NAC neoadjuvant chemotherapy, OR odds ratio, 95\% CI 95\% confidence interval *The $P$ value is significant

demonstrated in TILs evaluation. However, large-scale investigation should be performed to assess the intra- and inter-observer reproducibility of TILs evaluation before the application of TILs assessment in clinical practice.

Several studies have evaluated the predictive value of sTILs and iTILs for pCR in neoadjuvant treated TNBCs [12, 21, 29-32]. Khoury et al. found that both sTILs and iTILs were independent predictors for $\mathrm{pCR}$ in TNBCs
[33]. Denkert et al's study revealed that iTILs was a significant independent parameter for $\mathrm{pCR}$ in breast cancers in both training and validation cohorts, while sTILs was a strong predictor for pCR just in validation cohort [9]. The study of Issa-Nummer et al. showed that, in HER2-negative breast cancer, sTILs was a significant independent predictor for $\mathrm{pCR}$ in multivariate analysis, while iTILs was significant for pCR only in univariate

Table 4 Correlations between TILs and pCR in clinicopathologic subgroups

\begin{tabular}{|c|c|c|c|c|c|c|c|}
\hline \multirow[t]{2}{*}{ Variable } & \multirow[t]{2}{*}{ No. of patients } & \multicolumn{3}{|c|}{ Per 10\% sTILs increase } & \multicolumn{3}{|c|}{ Per 10\% iTlLs increase } \\
\hline & & Adjusted OR & $95 \% \mathrm{Cl}$ & $P$-value* & Adjusted OR & $95 \% \mathrm{Cl}$ & $P$-value* \\
\hline \multicolumn{8}{|l|}{ Age (years) } \\
\hline$<50$ & 80 & 1.06 & $1.01-1.11$ & 0.78 & 1.09 & $1.01-1.18$ & 0.64 \\
\hline$\geq 50$ & 86 & 1.07 & $1.02-1.12$ & & 1.12 & $1.03-1.22$ & \\
\hline \multicolumn{8}{|l|}{ Tumor size $(\mathrm{cm})$} \\
\hline$\leq 2$ & 22 & 1.05 & $0.99-1.12$ & 0.68 & 1.07 & $0.92-1.25$ & 0.72 \\
\hline $2-5$ & 115 & 1.06 & $1.02-1.10$ & & 1.09 & $1.02-1.16$ & \\
\hline$>5$ & 29 & 1.11 & $1.00-1.24$ & & 1.16 & $1.00-1.35$ & \\
\hline \multicolumn{8}{|l|}{ Lymph node status } \\
\hline Negative & 53 & 1.07 & $1.00-1.14$ & 0.81 & 1.07 & $0.96-1.18$ & 0.56 \\
\hline Positive & 113 & 1.06 & $1.02-1.10$ & & 1.11 & $1.04-1.19$ & \\
\hline \multicolumn{8}{|l|}{ Grade } \\
\hline 2 & 31 & 1.01 & $0.90-1.13$ & 0.34 & 1.02 & $0.84-1.23$ & 0.46 \\
\hline 3 & 135 & 1.07 & $1.03-1.10$ & & 1.11 & $1.04-1.17$ & \\
\hline \multicolumn{8}{|l|}{ LVI } \\
\hline Negative & 130 & 1.06 & $1.02-1.10$ & 0.49 & 1.08 & $1.02-1.15$ & 0.84 \\
\hline Positive & 36 & 1.14 & $0.93-1.39$ & & 1.11 & $0.83-1.48$ & \\
\hline \multicolumn{8}{|l|}{ NAC } \\
\hline Anthracycline + paclitaxel & 136 & 1.07 & $1.03-1.12$ & 0.23 & 1.12 & $1.06-1.20$ & 0.14 \\
\hline Paclitaxel + platinum & 30 & 1.01 & $0.93-1.10$ & & 1.00 & $0.87-1.14$ & \\
\hline
\end{tabular}

Abbreviations: $p C R$ pathological complete response, sTILs stromal tumor-infiltrating lymphocytes, iTILs intratumoral tumor-infiltrating lymphocytes, LVI lymphovascular invasion, NAC neoadjuvant chemotherapy, OR odds ratio, 95\% Cl 95\% confidence interval

*The chi-squared test 

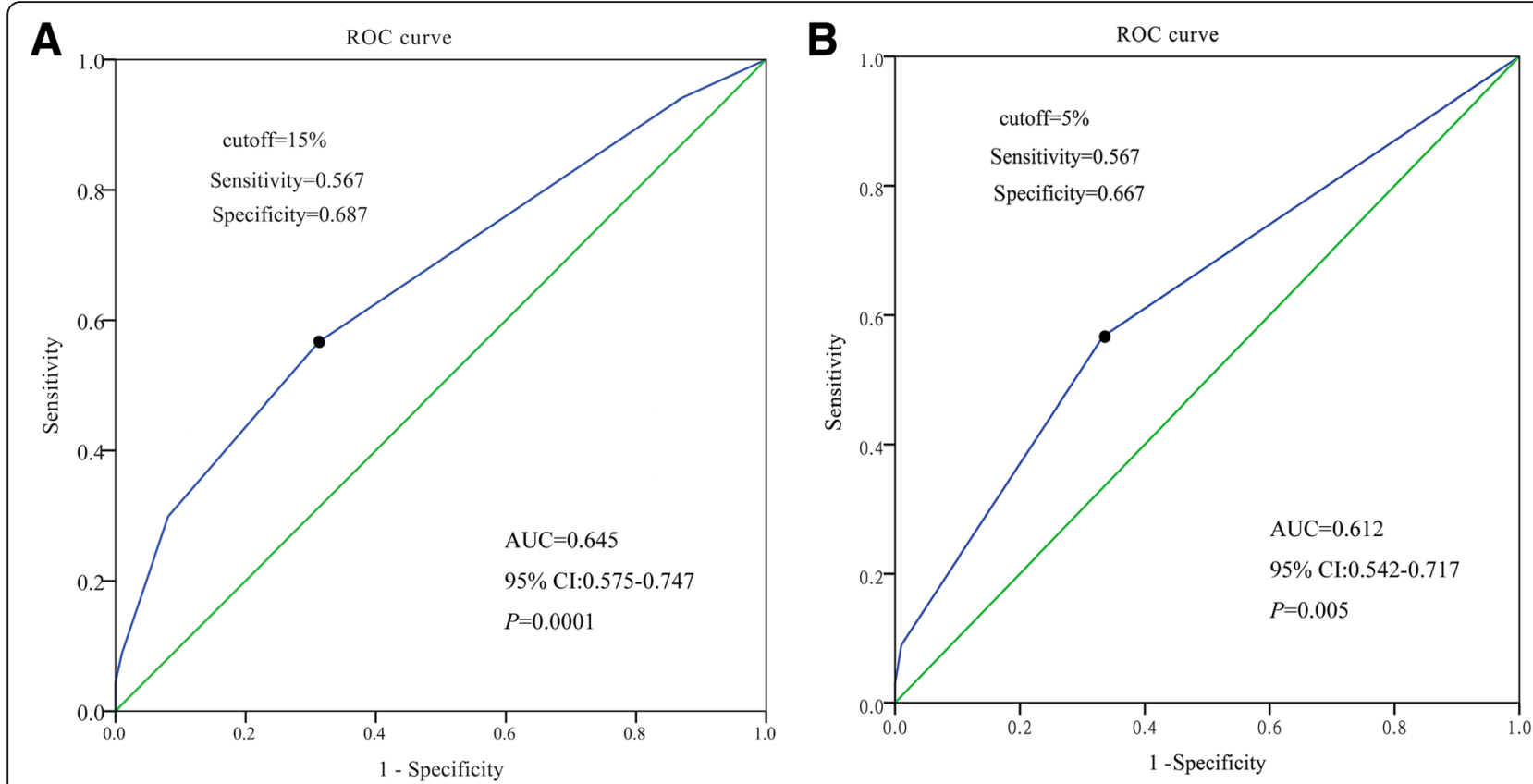

Fig. 3 Receiver operating characteristics (ROC) analysis for the thresholds of TILs to predict PCR in neoadjuvant treated TNBCs. ROC curves of sTILs (a) and iTILs (b). The black dot indicated the optimal threshold. The area under the curve (AUC), 95\% confidence interval (CI) and $P$-value were listed in the picture

but not in multivariate analysis [34]. Our study indicated that both higher sTILs and iTILs score strongly predicted higher $\mathrm{pCR}$ rate in univariate analysis, and both sTILs and iTILs score were independent predictors for $\mathrm{PCR}$ in multivariate analysis in TNBCs.

The cutoff value of TILs to predict therapeutic response has been analyzed in previous studies. Some studies found that lymphocyte-predominant breast cancer (LPBC, defined as involving $\geq 50 \%$ or $\geq 60 \%$ lymphocytic infiltration of either tumor stroma or cell nests) was an independent predictor of $\mathrm{pCR}$ for neoadjuvant treated triple-negative and HER2-positive breast cancers [22, 34-36]. However, the 2014 International TILs Working Group recommendations suggested that it was arbitrary to define $50-60 \%$ as the threshold for LPBC, because of the relatively low proportion of these cases in breast cancers [14]. In our previous study, only $3.5 \%$ of TNBCs had more than $50 \%$ lymphocytes [27]. In this study, only 7 of 166 TNBCs (4.2\%) had more than $50 \%$ lymphocyte infiltration in core needle biopsy specimens. It was unsuitable to define a cutoff value of $50 \%$ as LPBC to predict PCR because of the limited clinical implication caused by the low proportion of these cases. In our study, ROC curve analysis revealed that $20 \%$ threshold of sTILs and $10 \%$ threshold of iTILs may be more optimal to predict pCR compared with other cutoff values. TNBCs with more than $20 \%$ sTILs or with more than $10 \%$ iTILs were associated with higher pCR rate in

Table 5 Comparisons between different thresholds of sTILs and iTILS

\begin{tabular}{clllllll}
\hline Threshold (\%) & No. of patients (\%) & Sensitivity (\%) & Specificity (\%) & PPV (\%) & NPV (\%) & Accuracy (\%) & Youden's Index \\
\hline STILs & & & & & & & \\
20 & 41.6 & 56.7 & 68.7 & 55.1 & 70.1 & 63.9 & 0.254 \\
50 & 4.2 & 9.0 & 99.0 & 85.7 & 61.6 & 62.7 & 0.08 \\
60 & 1.8 & 4.5 & 100.0 & 100.0 & 60.7 & 61.4 & 0.045 \\
ITILS & & & & & 62.7 & 0.234 \\
10 & 42.8 & 56.7 & 66.7 & 53.5 & 69.5 & 6.7 & 0.078 \\
20 & 4.2 & 8.9 & 98.9 & 85.7 & 61.6 & 62.7 & 0.029 \\
30 & 1.2 & 2.9 & 100.0 & 100.0 & 60.4 & 60.8 &
\end{tabular}

Abbreviations: sTILs stromal tumor-infiltrating lymphocytes, iTILs intratumoral tumor-infiltrating lymphocytes, PPV positive predictive value, NPV negative predictive value, Youden's Index = sensitivity + specificity - 1

${ }^{\mathrm{a}} \mathrm{PPV}=\mathrm{pCR}$ rate 
Table 6 Correlations between the optimal thresholds of TILs and pCR in neoadjuvant treated TNBCs

\begin{tabular}{|c|c|c|c|c|c|c|}
\hline \multirow[t]{2}{*}{ Variables } & \multicolumn{3}{|c|}{ Univariate analysis } & \multicolumn{3}{|c|}{ Multivariate analysis } \\
\hline & $\mathrm{OR}$ & $95 \% \mathrm{Cl}$ & $P$-value & $\mathrm{OR}$ & $95 \% \mathrm{Cl}$ & $P$-value \\
\hline ITILs (<10\% vs. $\geq 10 \%)$ & 2.62 & $1.38-4.97$ & $0.003^{*}$ & 1.97 & $0.98-3.98$ & 0.06 \\
\hline STILs (<20\% vs. $\geq 20 \%)$ & 2.87 & $1.51-5.47$ & $0.001^{*}$ & 2.85 & $1.38-5.90$ & $0.005^{*}$ \\
\hline Age(years) (<50 vs. $\geq 50)$ & 0.51 & $0.27-0.95$ & $0.04^{*}$ & 0.70 & $0.33-1.45$ & 0.33 \\
\hline Histological grade (2 vs. 3) & 2.74 & $1.11-6.80$ & $0.03^{*}$ & 1.02 & $0.34-3.10$ & 0.97 \\
\hline Tumor size $(\mathrm{cm})(\leq 2$ vs. $2-5$ vs. $>5)$ & 1.30 & $0.74-2.29$ & 0.37 & 1.12 & $0.58-2.14$ & 0.74 \\
\hline Nodal status (positive vs. negative) & 0.93 & $0.48-1.81$ & 0.84 & 1.76 & $0.82-3.79$ & 0.15 \\
\hline LVI (positive vs. negative) & 0.06 & $0.01-0.26$ & $0.0001^{*}$ & 0.05 & $0.01-0.25$ & $0.0001^{*}$ \\
\hline Ki-67 index & 2.47 & $1.17-5.24$ & $0.02^{*}$ & 1.03 & $1.01-1.04$ & $0.004^{*}$ \\
\hline NAC (Anthracycline + paclitaxel vs. paclitaxel + platinum) & 0.83 & $0.37-1.87$ & 0.65 & 0.99 & $0.49-2.00$ & 0.97 \\
\hline
\end{tabular}

Abbreviations: $p C R$ pathological complete response, TNBCs triple-negative breast cancers, sTILs stromal tumor-infiltrating lymphocytes, iTILs intratumoral tumorinfiltrating lymphocytes, LVI lymphovascular invasion, NAC neoadjuvant chemotherapy, OR odds ratio, 95\% CI 95\% confidence interval *The $P$ value is significant

univariate analysis. A $20 \%$ threshold of sTILs was an independent predictor for $\mathrm{pCR}$ in multivariate analysis. However, a 10\% threshold of iTILs wasn't an independent predictor for $\mathrm{pCR}$ in multivariate analysis, which may due to the relatively low score of iTILs in our study. In addition, our study performed a predictive model for therapeutic response by the combination of sTILs score, patients' age, histological grade, LVI and Ki-67 index in TNBCs. It was shown that the combined five variables had relatively high sensitivity and specificity. Therefore, the predictive model might have potential value to predict NAC treated response in TNBCs in practice.

\section{ROC Curve}

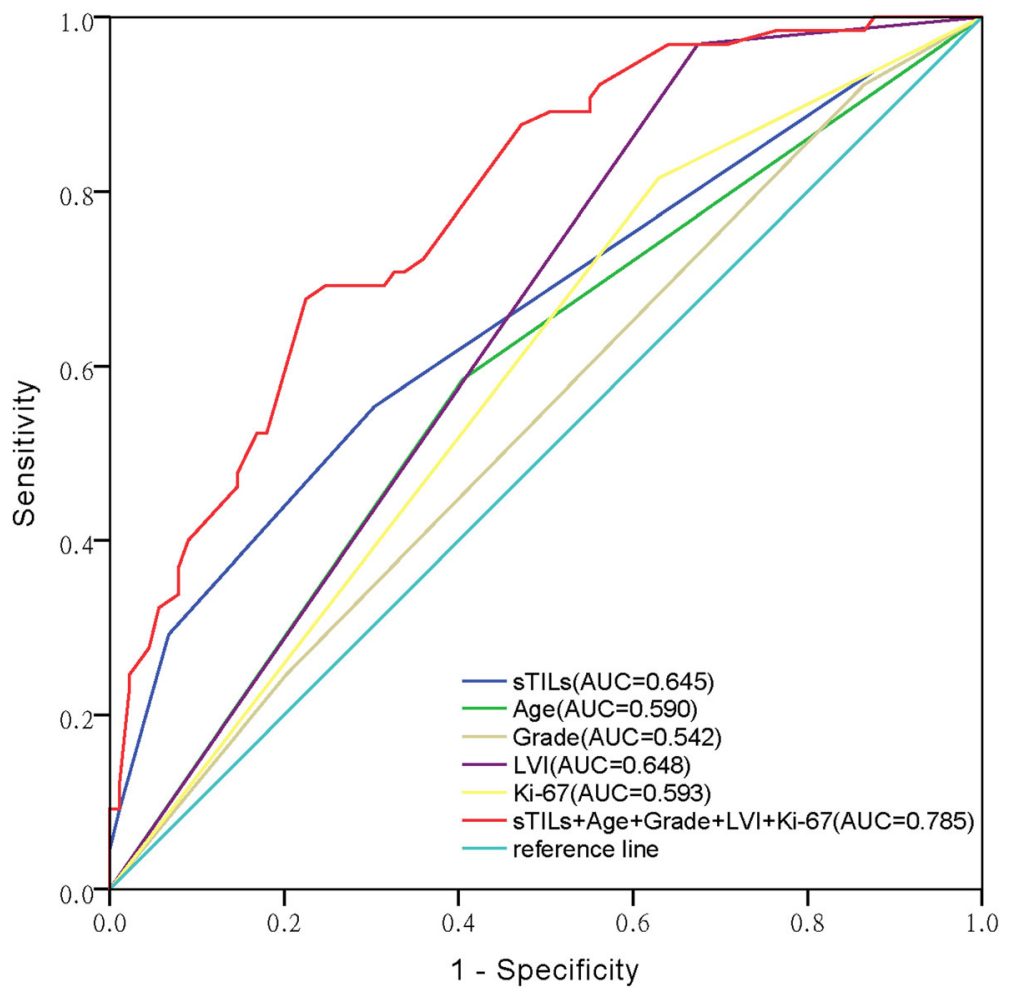

Fig. 4 Receiver operating characteristics (ROC) analysis for the combination of sTILs with clinicopathologic parameters (patients' age, histological grade, LVI and Ki-67 index) to predict pCR in TNBCs. The AUC (area under the curve) were listed in the picture. 95\% confidence interval (Cl) of combined predictive model was $0.714-0.856(P=0.0001)$, sTILs: $95 \% \mathrm{Cl} 0.575-0.747(P=0.0001)$, patients' age: $95 \% \mathrm{Cl} 0.499-0.681(P=0.057)$, histological grade: $95 \% \mathrm{Cl} 0.450-0.634(P=0.373)$, LVI: 95\% Cl 0.562-0.733 ( $P=0.002)$, Ki-67: 95\% Cl 0.503-0.683 ( $P=0.049)$ 
The relationships between clinicopathologic parameters and TILs were also analyzed in our study. It was revealed that both higher scores of sTILs and iTILs were related with higher Ki-67 index and higher histological grade, and higher iTILs scores were positively correlated with negative LVI in TNBCs. Krishnamurti et al. found that TILs was significantly associated with histologic grade 3 in TNBCs [37]. The study of Chung et al. showed that infiltration of CD4+, CD8+, and FOXP3+ TILs was significantly higher in tumors with high Ki-67 index [38]. Pan et al.'s study revealed that the percentage of sTILs and density of CD8+ T-lymphocytes were positively correlated with Ki-67 in TNBCs [39]. Lee et al. found that TNBCs with higher levels of TILs showed lower LVI [40]. However, the relationships between clinicopathologic characteristics and TILs still need to be further explored.

The relationship between TILs subpopulations and therapeutic response has been studied in breast cancers in recent years. Garcia-Martinez et al. and Castaneda et al. found that higher ratio of $\mathrm{CD} 8+/ \mathrm{CD} 4+$ was associated with higher $\mathrm{pCR}$ rate in pre-NAC breast cancers $[11,12]$. The study of Seo et al. revealed that CD8+ TILs was an independent predictor for $\mathrm{pCR}$ irrespective of breast cancer subtypes [41]. The research of Asano et al. showed that the $\mathrm{pCR}$ rate was significantly higher in the high CD8 +/FOXP3+ TIL ratio (CFR) group, and high-CFR status was an independent predictor of a favorable prognosis for TNBC and HER2+ breast cancer [42]. However, the scoring methods of subgroup evaluation were not standardized in these studies, the clinical application of TILs subpopulations still needs more available evidence.

\section{Conclusions}

In summary, our study indicated that TILs scored by methods recommended by International TILs Working Group 2014 in pre-NAC core needle biopsy specimens was significantly correlated with $\mathrm{pCR}$ in TNBCs, higher TILs scores predicting higher pCR rate. Both sTILs and iTILs were independent predictors for $\mathrm{pCR}$ in TNBCs. A $20 \%$ threshold for sTILs may be feasible to predict pCR to NAC in TNBCs. The combination of sTILs and other clinicopathologic variables might have potential value to predict NAC treated response in TNBCs in practice.

\section{Abbreviations}

ER: estrogen receptor; HER2: human epidermal growth factor receptor 2; LPBC: lymphocyte-predominant breast cancer; PCR: pathological complete response; PgR: progesterone receptor; TILs: tumor-infiltrating lymphocytes; TNBCs: triple-negative breast cancers

\section{Funding}

This work was supported by grants from Research Project of the Science and Technology Commission of Shanghai Municipality (Project No: 15411965100, for Ruohong Shui).

\section{Availability of data and materials}

The data are available upon request on the following e-mail address: 15211230043@fudan.edu.cn.

\section{Authors' contributions}

Work design (all authors). Data Collection and Drafting the article (M.R). Data statistics and analysis (T.T). Revision of the article (R.S). All authors read and approved the final manuscript.

\section{Ethics approval and consent to participate}

The study was approved by Ethics Institutional Review Board of Fudan University Shanghai Cancer Center. The patients' records/information were anonymized and de-identified prior to analysis.

\section{Consent for publication}

Not applicable.

\section{Competing interests}

The author declares that they have no competing interests.

\section{Publisher's Note}

Springer Nature remains neutral with regard to jurisdictional claims in published maps and institutional affiliations.

Received: 26 June 2018 Accepted: 20 August 2018

Published online: 31 August 2018

\section{References}

1. Podo F, Buydens LM, Degani H, Hilhorst R, Klipp E, Gribbestad IS, et al. Triple-negative breast cancer: present challenges and new perspectives. Mol Oncol. 2010:4:209-29.

2. Dent R, Trudeau M, Pritchard KI, Hanna WM, Kahn HK, Sawka CA, et al. Triple-negative breast cancer: clinical features and patterns of recurrence. Clin Cancer Res. 2007;13:4429-34.

3. Liedtke C, Mazouni C, Hess KR, Andre F, Tordai A, Mejia JA, et al. Response to neoadjuvant therapy and long-term survival in patients with triplenegative breast cancer. J Clin Oncol. 2008;26:1275-81.

4. Kumar P, Aggarwal R. An overview of triple-negative breast cancer. Arch Gynecol Obstet. 2016;293:247-69.

5. Stagg J, Allard B. Immunotherapeutic approaches in triple-negative breast cancer: latest research and clinical prospects. Ther Adv Med Oncol. 2013;5: 169-81.

6. van Rooijen JM, Stutvoet TS, Schroder CP, de Vries EG. Immunotherapeutic options on the horizon in breast cancer treatment. Pharmacol Ther. 2015; 156:90-101

7. Fisher CS, Ma CX, Gillanders WE, Aft RL, Eberlein TJ, Gao F, et al. Neoadjuvant chemotherapy is associated with improved survival compared with adjuvant chemotherapy in patients with triple-negative breast cancer only after complete pathologic response. Ann Surg Oncol. 2012;19:253-8.

8. Kong X, Moran MS, Zhang N, Haffty B, Yang Q. Meta-analysis confirms achieving pathological complete response after neoadjuvant chemotherapy predicts favourable prognosis for breast cancer patients. Eur J Cancer. 2011; 47:2084-90

9. Denkert C, Loibl S, Noske A, Roller M, Muller BM, Komor M, et al. Tumorassociated lymphocytes as an independent predictor of response to neoadjuvant chemotherapy in breast cancer. J Clin Oncol. 2010;28:105-13.

10. Jung YY, Hyun CL, Jin MS, Park IA, Chung YR, Shim B, et al. Histomorphological factors predicting the response to neoadjuvant chemotherapy in triplenegative breast Cancer. J Breast Cancer. 2016:19:261-7.

11. Garcia-Martinez E, Gil GL, Benito AC, Gonzalez-Billalabeitia E, Conesa MA, Garcia Garcia T, et al. Tumor-infiltrating immune cell profiles and their change after neoadjuvant chemotherapy predict response and prognosis of breast cancer. Breast Cancer Res. 2014;16:488.

12. Castaneda CA, Mittendorf E, Casavilca S, Wu Y, Castillo M, Arboleda P, et al. Tumor infiltrating lymphocytes in triple negative breast cancer receiving neoadjuvant chemotherapy. World J Clin Oncol. 2016;7:387-94.

13. Loi S, Sirtaine N, Piette F, Salgado R, Viale G, Van Eenoo F, et al. Prognostic and predictive value of tumor-infiltrating lymphocytes in a phase III randomized adjuvant breast cancer trial in node-positive breast cancer comparing the addition of docetaxel to doxorubicin with doxorubicinbased chemotherapy: BIG 02-98. J Clin Oncol. 2013;31:860-7. 
14. Salgado R, Denkert C, Demaria S, Sirtaine N, Klauschen F, Pruneri G, et al. The evaluation of tumor-infiltrating lymphocytes (TILs) in breast cancer: recommendations by an international TILs working group 2014. Ann Oncol. 2015;26:259-71.

15. Wolff AC, Hammond ME, Schwartz JN, Hagerty KL, Allred DC, Cote RJ, et al. American Society of Clinical Oncology/College of American Pathologists guideline recommendations for human epidermal growth factor receptor 2 testing in breast cancer. J Clin Oncol. 2007;25:118-45.

16. Hammond ME, Hayes DF, Wolff AC, Mangu PB, Temin S. American society of clinical oncology/college of american pathologists guideline recommendations for immunohistochemical testing of estrogen and progesterone receptors in breast cancer. J Oncol Pract. 2010;6:195-7.

17. Lakhani SR, Ellis IO, Schnitt SJ, Tan PH, van de Vijver MJ. WHO Classification of Tumours of the Breast. Lyon: IARC. 2012;4.

18. Bloom HJ, Richardson WW. Histological grading and prognosis in breast cancer; a study of 1409 cases of which 359 have been followed for 15 years. Br J Cancer. 1957;11:359-77.

19. Elston CW, Ellis IO. Pathological prognostic factors in breast cancer. I. The value of histological grade in breast cancer: experience from a large study with long-term follow-up. Histopathology. 1991;19:403-10.

20. Ogston KN, Miller ID, Payne S, Hutcheon AW, Sarkar TK, Smith I, et al. A new histological grading system to assess response of breast cancers to primary chemotherapy: prognostic significance and survival. Breast (Edinburgh, Scotland). 2003;12:320-7

21. Ono M, Tsuda H, Shimizu C, Yamamoto S, Shibata T, Yamamoto H, et al. Tumor-infiltrating lymphocytes are correlated with response to neoadjuvant chemotherapy in triple-negative breast cancer. Breast Cancer Res Treat. 2012;132:793-805.

22. Denkert C, Loibl S, Salat C, Sinn B, Schem C, Endris V, et al. Abstract S1-06: Increased tumor-associated lymphocytes predict benefit from addition of carboplatin to neoadjuvant therapy for triple-negative and HER2-positive early breast cancer in the GeparSixto trial (GBG 66). Cancer Res. 2013;73:S1-06-S01-06.

23. Loi S, Michiels S, Salgado R, Sirtaine N, Jose V, Fumagalli D, et al. Tumor infiltrating lymphocytes are prognostic in triple negative breast cancer and predictive for trastuzumab benefit in early breast cancer: results from the FinHER trial. Ann Oncol. 2014:25:1544-50.

24. Adams S, Gray RJ, Demaria S, Goldstein L, Perez EA, Shulman LN, et al. Prognostic value of tumor-infiltrating lymphocytes in triple-negative breast cancers from two phase III randomized adjuvant breast cancer trials: ECOG 2197 and ECOG 1199. J Clin Oncol. 2014;32:2959-66.

25. Pruneri G, Vingiani A, Bagnardi V, Rotmensz N, De Rose A, Palazzo A, et al. Clinical validity of tumor-infiltrating lymphocytes analysis in patients with triple-negative breast cancer. Ann Oncol. 2016;27:249-56.

26. Park HS, Heo I, Kim JY, Kim S, Nam S, Park S, et al. No effect of tumorinfiltrating lymphocytes (TILs) on prognosis in patients with early triplenegative breast cancer: validation of recommendations by the international TILs working group 2014. J Surg Oncol. 2016;114:17-21.

27. Tian T, Ruan M, Yang W, Shui R. Evaluation of the prognostic value of tumor-infiltrating lymphocytes in triple-negative breast cancers. Oncotarget. 2016;7:44395-405.

28. Swisher SK, Wu Y, Castaneda CA, Lyons GR, Yang F, Tapia C, et al. Interobserver agreement between pathologists assessing tumor-infiltrating lymphocytes (TILs) in breast Cancer using methodology proposed by the international TILs working group. Ann Surg Oncol. 2016;23:2242-8.

29. Mao Y, Qu Q, Zhang Y, Liu J, Chen $X$, Shen $K$. The value of tumor infiltrating lymphocytes (TLLs) for predicting response to neoadjuvant chemotherapy in breast cancer: a systematic review and meta-analysis. PLoS One. 2014;9:e115103.

30. Wang $K, X u$ J, Zhang T, Xue D. Tumor-infiltrating lymphocytes in breast cancer predict the response to chemotherapy and survival outcome: a meta-analysis. Oncotarget. 2016;7:44288-98.

31. Li XB, Krishnamurti U, Bhattarai S, Klimov S, Reid MD, O'Regan R, et al. Biomarkers predicting pathologic complete response to neoadjuvant chemotherapy in breast Cancer. Am J Clin Pathol. 2016;145:871-8.

32. Hida Al, Sagara Y, Yotsumoto D, Kanemitsu S, Kawano J, Baba S, et al. Prognostic and predictive impacts of tumor-infiltrating lymphocytes differ between triple-negative and HER2-positive breast cancers treated with standard systemic therapies. Breast Cancer Res Treat. 2016;158:1-9.

33. Khoury T, Nagrale V, Opyrchal M, Peng X, Wang D, Yao S. Prognostic significance of stromal versus Intratumoral infiltrating lymphocytes in different subtypes of breast Cancer treated with cytotoxic neoadjuvant chemotherapy: Applied immunohistochemistry \& molecular morphology : AIMM; 2017:1.

34. Issa-Nummer Y, Darb-Esfahani S, Loibl S, Kunz G, Nekljudova V, Schrader I, et al. Prospective validation of immunological infiltrate for prediction of response to neoadjuvant chemotherapy in HER2-negative breast cancer--a substudy of the neoadjuvant GeparQuinto trial. PLoS One. 2013;8:e79775.

35. Denkert C, von Minckwitz G, Brase JC, Sinn BV, Gade S, Kronenwett R, et al. Tumor-infiltrating lymphocytes and response to neoadjuvant chemotherapy with or without carboplatin in human epidermal growth factor receptor 2positive and triple-negative primary breast cancers. J Clin Oncol. 2015;33: 983-91.

36. Ingold Heppner B, Untch M, Denkert C, Pfitzner BM, Lederer B, Schmitt W, et al. Tumor-infiltrating lymphocytes: a predictive and prognostic biomarker in neoadjuvant-treated HER2-positive breast Cancer. Clin Cancer Res. 2016; 22:5747-54.

37. Krishnamurti U, Wetherilt CS, Yang J, Peng L, Li X. Tumor-infiltrating lymphocytes are significantly associated with better overall survival and disease-free survival in triple-negative but not estrogen receptor-positive breast cancers. Hum Pathol. 2017;64:7-12.

38. Chung YR, Kim HJ, Jang MH, Park SY. Prognostic value of tumor infiltrating lymphocyte subsets in breast cancer depends on hormone receptor status. Breast Cancer Res Treat. 2017;161:409-20.

39. Pan BJ, Ping GQ, Zhang WM, Wang C, Li HX, Zhang ZH. CD8 and FOXP3 expression in stromal tumor-infiltrating lymphocytes of triple-negative breast carcinomas: a clinicopathologic study. Zhonghua bing li xue za zhi. 2016:45:540-4.

40. Lee HJ, Park IA, Song $I H$, Shin SJ, Kim JY, Yu JH, et al. Tertiary lymphoid structures: prognostic significance and relationship with tumour-infiltrating lymphocytes in triple-negative breast cancer. J Clin Pathol. 2016;69:422-30.

41. Seo AN, Lee HJ, Kim EJ, Kim HJ, Jang MH, Lee HE, et al. Tumour-infiltrating CD8+ lymphocytes as an independent predictive factor for pathological complete response to primary systemic therapy in breast cancer. $\mathrm{Br} \mathrm{J}$ Cancer. 2013;109:2705-13.

42. Asano Y, Kashiwagi S, Goto W, Kurata K, Noda S, Takashima T, et al. Tumourinfiltrating CD8 to FOXP3 lymphocyte ratio in predicting treatment responses to neoadjuvant chemotherapy of aggressive breast cancer. $\mathrm{Br}$ J Surg. 2016;103:845-54
Ready to submit your research? Choose BMC and benefit from:
- fast, convenient online submission
- thorough peer review by experienced researchers in your field
- rapid publication on acceptance
- support for research data, including large and complex data types
- gold Open Access which fosters wider collaboration and increased citations
- maximum visibility for your research: over $100 \mathrm{M}$ website views per year
At $\mathrm{BMC}$, research is always in progress.
Learn more biomedcentral.com/submissions 